\title{
ON THE IDEAL STRUCTURE OF THE ALGEBRA OF RADIAL FUNCTIONS
}

\author{
ALAN SCHWARTZ ${ }^{1}$
}

Abstract. Let $L$ denote the convolution Banach algebra of integrable functions defined on $R^{n}$ and let $L_{r}$ consist of the subalgebra of radial functions. If $I$ is a closed ideal of $L$, the zero-set of $I$ is defined by $Z(I)=\{y \mid \hat{f}(y)=0$ for all $f \in I\}$ where $\hat{f}$ is the Fourier transform of $f$. The following theorem is proved. If $I_{1}$ and $I_{2}$ are closed ideals of $L_{r}$ such that $I_{1} \subset I_{2}$ ( $\subset$ denotes proper inclusion) then there is a closed ideal $I$ such that $I_{1} \subset I \subset I_{2}$.

Let $n$ be a fixed positive integer, and let $L$ denote the Banach algebra of integrable functions defined on $\boldsymbol{R}^{n}$ with the usual norm and convolution. (The practice of identifying two functions which agree almost everywhere will be followed.) A function $f$ defined on $R^{n}$ is said to be radial if $f(\mathbf{x})=\phi(|\mathbf{x}|)$ for some function $\phi$ defined on $[0, \infty)$ and for almost every $\mathrm{x}$ in $R^{n} ; L_{r}$ will denote the space of radial functions contained in $L$. A function in $L$ is radial if and only if its Fourier transform is a radial function (see [1, pp. 69-79]), so $L_{r}$ is a Banach algebra. If $I$ is a closed ideal of $L$ or of $L_{r}$, let

$$
Z(I)=\{\boldsymbol{y} \mid \hat{f}(\mathbf{y})=0 \text { for every } f \in I\} .
$$

$Z(I)$ is called the zero-set of $I$.

Helson showed in [2] that if $I_{1}$ and $I_{2}$ are closed ideals of $L$ such that $Z\left(I_{1}\right)=Z\left(I_{2}\right)$ and $I_{1} \subset I_{2}$ ( $\subset$ denotes proper inclusion), then there is a closed ideal $I$ such that $I_{1} \subset I \subset I_{2}$. In the present paper Helson's theorem will be used to prove the following:

THEOREM. If $I_{1}$ and $I_{2}$ are closed ideals of $L_{r}$ such that $Z\left(I_{1}\right)=Z\left(I_{\mathbf{2}}\right)$ and $I_{1} \subset I_{2}$, then there is a closed ideal I of $L_{r}$ such that $I_{1} \subset I \subset I_{2}$.

The proof of the theorem will be given later; it is necessary, first, to examine how $L_{r}$ sits in $L$.

Let $d \mu$ be the positive measure of unit mass distributed uniformly on the hypersphere $S=\left\{x \mid x \in R^{n}\right.$ and $\left.|x|=1\right\}$, and set

Received by the editors September 5, 1969.

AMS 1969 subject classifications. Primary 4240, 4258.

Key words and phrases. Convolution algebra, Fourier transform, ideal structure, radial functions, zero-sets.

${ }^{1}$ Supported by an Assistant Professor Research Grant at the University of Missouri, St. Louis. 


$$
f_{r}(\mathbf{x})=\int f(|\mathbf{x}| \boldsymbol{y}) d \mu(\boldsymbol{y})
$$

The integral must exist for almost every $x$ by Fubini's theorem since $\boldsymbol{R}^{n}$ can be thought of as a product of two measure spaces: one being $S$ with the measure $d \mu$ and the other being $[0, \infty)$ with the measure $c \rho^{n-1} d \rho$ where $d \rho$ is Lebesgue measure and $c$ is the surface area of $S$. It also follows from Fubini's theorem that $f_{r}$ is in $L$. Define

$$
L_{0}=\left\{f \mid f \in L \text { and } f_{r}(x)=0 \text { for almost every } \mathbf{x} \text { in } \boldsymbol{R}^{n}\right\} ;
$$

finally let $f_{0}(\mathbf{x})=f(\mathbf{x})-f_{r}(\mathbf{x})$. The following lemmas list some properties of $L_{r}, L_{0}, f_{r}$, and $f_{0}$.

LEMMA 1. The map $f \rightarrow f_{r}$ is a continuous projection with unit norm, hence its null space $L_{0}$ is closed and so $L=L_{0} \oplus L_{r}$.

The proof of Lemma 1 follows from the easily verified facts that $\left\|f_{r}\right\| \leqq\|f\|$ and that $f=f_{r}$ if $f$ is radial.

A thorough discussion of this decomposition can be found in [3].

LEMMA 2. $f$ is contained in $L_{0}$ if and only if

$$
\int \hat{f}(\rho y) d \mu(y)=0 \quad(\rho>0) .
$$

Proof. Application of Fubini's theorem yields

$$
\begin{aligned}
\int \hat{f}(\rho y) d \mu(\boldsymbol{y}) & =\int_{R^{\mathbf{n}}} f(\mathbf{x}) d \mathbf{x}\left\{\int \exp (i \mathbf{x} \cdot \rho \mathbf{y}) d \mu(\mathbf{y})\right\} \\
& =\int_{R^{\mathbf{n}}} f(\mathbf{x}) K(\mathbf{x}) d x
\end{aligned}
$$

where $K(x)$ is the value of the inner integral. $K(x)$ is a radial function because $\mu$ is a weak limit of radial functions and $K(x)$ is the Fourier-Stieltjes transform of $\mu$, or see [1, pp. 69-79]. Conversion of the last in tegral into hyperspherical coordinates yields (2).

To prove the converse, suppose (2) holds for some $f$ in $L$. Then

$$
\int \hat{f}_{r}(|\mathbf{x}| \boldsymbol{y}) d \mu(\boldsymbol{y})+\int \hat{f}_{0}(|\mathbf{x}| \mathbf{y}) d \mu(\boldsymbol{y})=0
$$

The second integral vanishes by the first part of this lemma since $f_{0}$ is in $L_{0}$, and the value of the first integral is $\hat{f}_{r}(x)$. Thus $\hat{f}_{r}=0$, so $f_{r}=0$ and hence $f$ is in $L_{0}$. 
LEMMA 3. The convolution of a function in $L_{0}$ and a function in $L_{r}$ is contained in $L_{0}$.

Proof. Suppose $f$ is in $L_{r}$ and $g$ is in $L_{0}$. Then for each $\mathrm{x}$ in $R^{n}$

$$
\begin{aligned}
\int(f * g)^{\wedge}(|\mathbf{x}| \boldsymbol{y}) d \mu(\mathbf{y}) & =\int \hat{f}(|\mathbf{x}| \mathbf{y}) \hat{g}(|\mathbf{x}| \mathbf{y}) d \mu(\mathbf{y}) \\
& =\hat{f}(\mathbf{x}) \int \hat{g}(|\mathbf{x}| \boldsymbol{y}) d \mu(\mathbf{y}) \\
& =0
\end{aligned}
$$

by Lemma 2 .

Lemma 4. Let $I$ be a closed ideal of $L_{r}$ and let $K$ be the closed ideal of $L$ generated by $I$. Then $I=K \cap L_{r}$ and $Z(I)=Z(K)$.

Proof. $I$ is contained in $K$, hence in $K \cap L_{r}$. The fact that $K \cap L_{r}$ is contained in $I$ will follow from the stronger fact that if $f$ is in $K$, then $f_{r}$ is in $I$. Suppose

$$
f=h+\sum_{i=1}^{m} h_{i} * g_{i} \quad\left(h \in I, h_{i} \in I, g_{i} \in L ; i=1,2, \cdots, m\right) .
$$

Then $f=h+\sum_{i=1}^{m} h_{i} *\left(g_{i}\right)_{r}+\sum_{i=1}^{m} h_{i} *\left(g_{i}\right)_{0}$. The second sum is contained in $L_{0}$ by Lemma 3 and the first sum is contained in $I$ because $I$ is an ideal of $L_{r}$. Finally, the first sum plus $h$ is $f_{r}$ by Lemma 1; hence if $f$ has the form (3) then $f_{r}$ is in $I$. If $f$ is any function in $K$, there is a sequence $\left\{f_{k}\right\}$ of finite linear combinations of the form of (3) such that $f_{k}$ converges to $f$ in $L$. The transformation of $f$ into $f_{r}$ is continuous on $L$ so $\left\{\left(f_{k}\right)_{r}\right\}$ converges to $f_{r}$. Since $I$ is closed, it must contain $f_{r}$.

Finally $Z(K) \subseteq Z(I)$ because $I \subseteq K$ and $Z(K)=Z(I)$ since finite linear combinations of the form of (3) are dense in $K$.

Proof of Theorem. Let $K_{1}$ and $K_{2}$ be the closed ideals of $L$ generated by $I_{1}$ and $I_{2}$ respectively. Then $K_{1} \subset K_{2}$ by Lemma 4 because $K_{1} \cap L_{r}=I_{1} \subset I_{2}=K_{2} \cap L_{r}$, and

$$
Z\left(K_{1}\right)=Z\left(I_{1}\right)=Z\left(I_{2}\right)=Z\left(K_{2}\right) .
$$

By Helson's theorem there must be a closed ideal $K$ such that $K_{1} \subset K \subset K_{2}$. Since $K_{2}$ is the ideal generated by $I_{2}$, it follows that $K \cap L_{r} \subset I_{2}$. The inclusion $I_{1} \subset K \cap L_{r}$ is not immediate. Suppose there is no closed ideal $K$ of $L$ such that

$$
I_{1} \subset K \cap L_{r} \subset I_{2}
$$


then define $\nVdash$ to be the collection of all closed ideals of $L$ such that

$$
K_{1} \subset K \subset K_{2} \text { and } K \cap L_{r}=I_{1} \text {. }
$$

Let $\Re$ be ordered by inclusion and let $K^{*}$ be the union of all the ideals in a maximal chain of $\mathcal{K}$.

$K^{*}$ is contained in $\nVdash$. To see this let $J$ be the closure in $L$ of $K^{*}$. If $f$ is in $K^{*} \cap L_{r}$, then $f$ is in $K \cap L_{r}$ for some $K$ in $\nVdash$ so $f$ is in $I_{1}$; thus $K^{*} \cap L_{r}=I_{1}$ so $J \cap L_{r}=I_{1}$. Since $J \cap L_{r}=I_{1}$, it follows that $J \subset K_{2}$. Since $K^{*}$ is a union of elements of $\varkappa$ it follows that $K_{1} \subset J \subset K_{2}$. Thus $J$ is in $\nVdash$ and so $K^{*}=J$ by the construction of $K^{*}$; hence, $K^{*}$ is in $\Re$. It also follows that $Z\left(K^{*}\right)=Z\left(K_{2}\right)$ because $K^{*}$ lies between $K_{1}$ and $K_{2}$.

Helson's theorem can now be invoked to guarantee the existence of an ideal $K^{* *}$ such that $K^{*} \subset K^{* *} \subset K_{2}$. Since $K^{* *} \subset K_{2}$ it follows that $K^{* *} \cap L_{r} \subset I_{2}$, and the proper inclusion $I_{1} \subset K^{* *} \cap L_{r}$ holds by the construction of $K^{*}$. Thus $K^{* *}$ contradicts our assumption that no ideal of $L$ satisfies (4).

\section{REFERENCES}

1. S. Bochner and K. Chandrasekharan, Fourier transforms, Ann. of Math. Studies, no. 19, Princeton Univ. Press, Princeton, N. J., 1949. MR 11, 173.

2. H. Helson, On the ideal structure of group algebras, Ark. Mat. 2 (1952), 83-86. MR 14, 246.

3. E. M. Stein and G. Weiss, An introduction to Fourier analysis in Euclidean spaces, Princeton Univ. Press, Princeton, N. J. (to appear).

4. Sadahiro Saeki, An elementary proof of a theorem of Henry Helson, Tohoku Math. J. (2) 20 (1968), 244-247. MR 37 \#6694.

University of Missouri, St. LouIs, Missouri 63121 